\title{
Underwater Image Enhancement Using Enhanced Detail \& Dehaze Technique (DDE)
}

\author{
Abdullah Bade ${ }^{1}$, Danny Ngo Lung Yao ${ }^{{ }^{*}}$ and Zarinah Waheed ${ }^{2}$ \\ ${ }^{1}$ Faculty of Science and Natural Resources (FSSA), University Malaysia Sabah (UMS), \\ Jalan UMS, 88400 Kota Kinabalu, Sabah, Malaysia \\ ${ }^{2}$ Borneo Marine Research Institute, University Malaysia Sabah (UMS), \\ Jalan UMS, 88400 Kota Kinabalu, Sabah, Malaysia
}

\begin{abstract}
Underwater images are often distorted by blurring, greenish appearance, noises, bright artifacts, nonuniform lighting, low contrast and limited range visibility. Thus, the underwater images need to be enhanced in order to restore the visibility and image quality. This paper proposes an Enhanced Detail \& Dehaze Technique (DDE) to restore the underwater image visibility. The proposed method removes the blurriness based on modified channel prior scheme and improves the image details based on enhanced Son method. Additionally, the image contrast is enhanced based on CLAHE. The MSE test and PSNR test are used to evaluate the effectiveness of the proposed technique on underwater image enhancement. From the conducted tests, the proposed method performed better in restoring the underwater image visibility compared the dark channel prior scheme.
\end{abstract}

Keywords: Underwater image enhancement, dark channel prior, Enhanced Detail \& Dehaze Technique (DDE)

\section{INTRODUCTION}

In this modern world, underwater computer vision had been developed to investigate the valuable resources such as rare deep-sea animals and corals. The accuracy of underwater computer vision is very reliable on underwater image quality. However, underwater images often suffered a various level of distortions due to the light absorption and scattering effects. Water tends to absorb a wide range of wavelength in the electromagnetic from the longest wavelength to shortest wavelength. This means that water will absorb more red light as compared with blue and green lights. Thus, the underwater image often results in the bluish or greenish appearance. Besides, the effects of underwater image distortion also included blurring, unsteady image noises, limited range visibility, bright artifacts, low contrast, and hazy effects. Therefore, this paper intends to enhance the underwater image quality and preserve the image details
Polarization analysis method (Schechner \& Karpel, 2005) is one of the early intentions to correct colour and improve visibility range and image contrast. Dark channel prior (DCP) (He et. al., 2009) which originally for haze removal purpose had been adapted for underwater image enhancement. A DCP with unsupervised colour correction method (Yang, et al., 2011) had enhanced the image quality and colour contrast. Later, an underwater DCP (UDCP) (Wen et. al., 2013) proposed an underwater optical model to consider the light absorption and light scattering effects on underwater image. In addition, a guided trigonometric bilateral filter had replaced the soft matting in DCP to reduce computational time and later an enhancement method based on automatic colour equalization had been used to improve the image contrast ( $\mathrm{Lu}$ et. al., 2013). Despite DCP origin on haze removal, DCP had proved its performance on underwater image enhancement is better than histogram equalization (HE) (Sahtya et. al., 2015). The DCP also proposed to apply on blue and green channels only 
where the red channel will used to correct colour based on Gray-World assumption (Li et. al., 2016). In contrast, red channel prior method (Borkar \& Bonde, 2016) had applied the DCP only on red channel as the red channel usually experienced maximum attenuation. Modified DCP method (Yao \& Xiang, 2018) obtained attenuations of RGB channels separately and applied colour correction. Contrast enhancement methods also being introduced for underwater image enhancement. Mixture Contrast Limited Adaptive Histogram Equalization (Mix-CLAHE) (Hitam et. al., 2013) combine the result of HSV-CLAHE and RGB-CLAHE by using Euclidean norm to improve the image visibility. In addition, RGB-CLAHE and HSVCLAHE had proved to have better performance in enhancing underwater image as compared with contrast stretching and HE (Wong et. al., 2014). Besides, CLAHE also improve the underwater image quality by fusing with unsharp mask (USM) (Zheng et. al., 2016).

\section{MATERIALS AND METHODS}

This paper proposed an enhanced detail and dehaze technique (DDE) for underwater image enhancement. Figure 1 shows the proposed method. The input underwater image $I c$ will be used to compute the modified channel Jmodified as equation (1) based on modified channel prior (Yao et. al., 2018) which stated that most images will consist of many dark pixels and bright pixels. The dark pixel and bright pixel refer to the pixel with the lowest intensity and highest intensity respectively. After computing the modified channels, median filter will be used to filter the modified channels. The background light $B$ will be estimated as the brightest pixel among the modified channels. Next, the transmission will be estimated as equation (2) where $\omega$ used to control the descattering effects. the underwater image model is defined as the equation (3) where $J$ is the descattered underwater image. From the equation (3), the descattered underwater image will be produced by using equation (4) where to is set as lower boundary of transmission. The image contrast of descattered underwater image will be improved using CLAHE.

$$
\begin{aligned}
& J^{\text {modified }}=\frac{\min _{c}\left(J^{c}\right)+\max _{c}\left(I^{c}\right)}{2} \\
& t=1-\omega\left(\frac{\text { median }\left(J^{\text {modified }}\right)}{B}\right) \\
& I=J t+B(1-t) \\
& J=\frac{I-B}{\max \left(t, t_{0}\right)}+B
\end{aligned}
$$

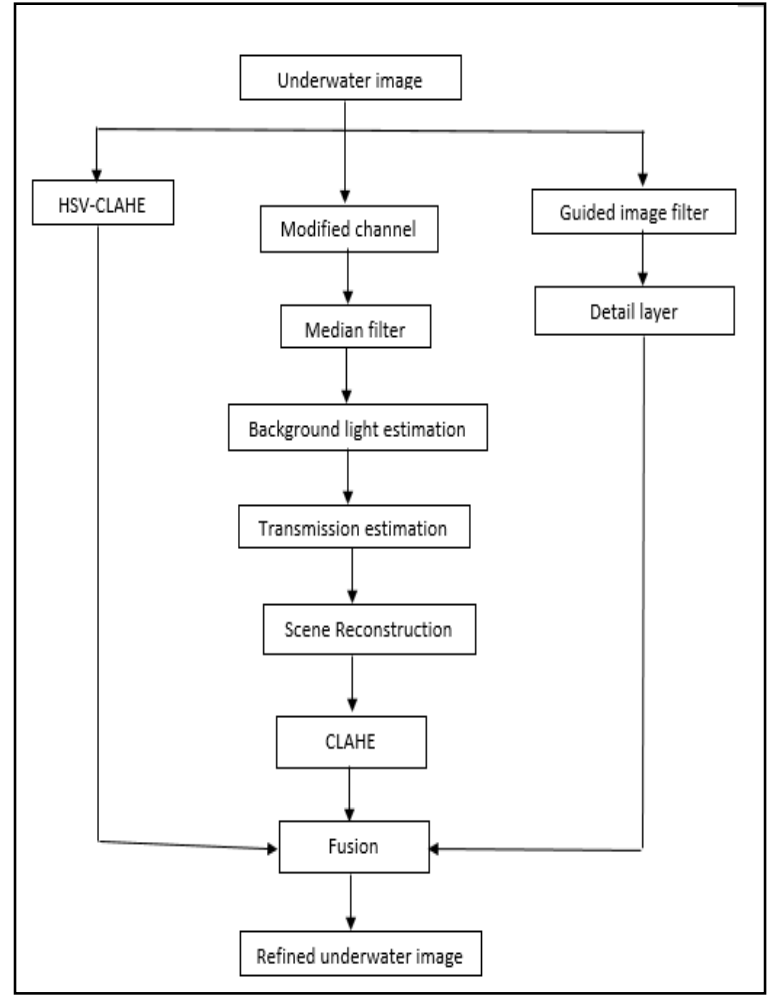

Figure 1. The proposed method.

Based on the Son method (Son et al., 2014), a digital image can be decomposed into base layer $S$ and detail layer $D$ as equation (5). First, we computed the base layer as the smooth version of input image by using guided image filtering. Then, the detail layer will be obtained as the equation (6). Meanwhile, the input image will undergo the HSV-CLAHE to produce IHSV-CLAHE improve the image contrast balance. At last, the descattered underwater image, HSV-CLAHE enhanced image and Detail layer will be combined through fusion as equation (7) to produce a refined underwater image where $n$ controls the scale of the details.

$$
\begin{aligned}
& I=S+D \\
& D=I-S \\
& I^{\text {refined }}=\frac{J+I^{H S V-C L A H E}}{2}+n D
\end{aligned}
$$

\section{RESULTS AND DISCUSSION}

There are five sample images used to test the effectiveness of Enhanced Detail \& Dehaze Technique (DDE) on underwater image enhancement. Table 1 shows the comparison between the DDE method and DCP method. From table 1, the proposed method showed a good result in increasing image 
visibility range, improve image details and remove the scattering effects. from the result of image 2, 4 and 5, the proposed method can remove the haze effects as the DCP method. From the image 1 and 3, proposed method can preserve more image details as compared with DCP.

Table 1. Comparison between proposed method and DCP

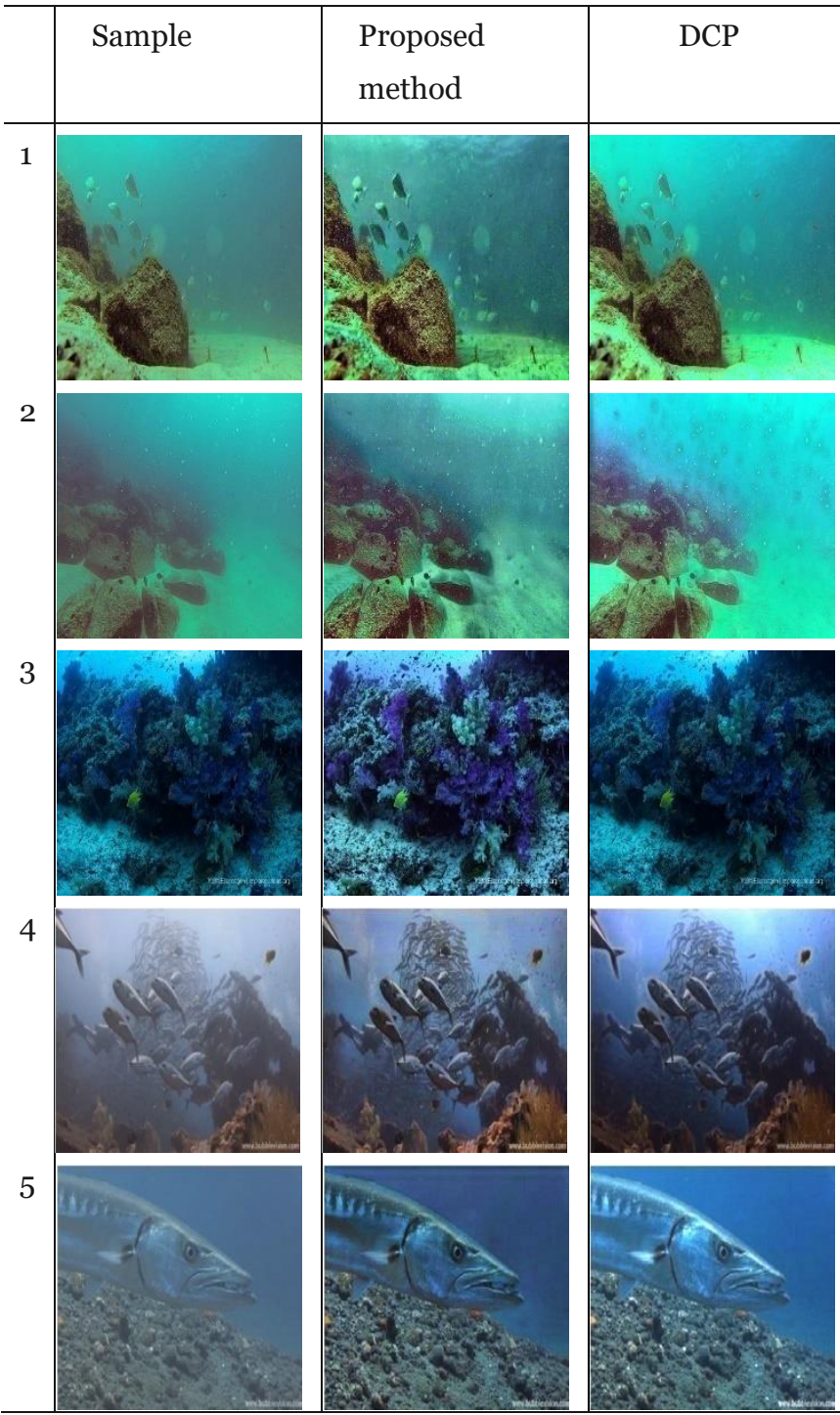

Mean Square Error (MSE) test is common test used to test the accuracy of the image contrast. The lower the MSE value obtained, the image contrast is more accurate. Table 2 shows the result of the proposed method and DCP method obtained in the MSE test. Overall, MSE test shows that the proposed method recovers the image contrast more accurate than the DCP method. However, the images with many tiny details such as sample 3 and 5 will cause the proposed method performed less accurate.
Table 2. MSE test

\begin{tabular}{l|rl}
\hline Sample & Proposed method & DCP \\
\hline 1 & 482.394 & 586,552 \\
2 & 465.943 & 1691.87 \\
3 & 1138.08 & 0.207328 \\
4 & 1019.05 & 1427.63 \\
5 & 696.545 & 610.971 \\
\hline
\end{tabular}

Peak-Signal-to-Noise-Ratio (PSNR) test is another common test to evaluate the amount of image noises contained in the image. Table 3 shows the result of proposed method and DCP method obtained in the PSNR test. The higher the value obtained in the PSNR test, the lower the amount of the image noises. The proposed method averagely reduces more image noises as compared with DCP as the PSNR values obtained are higher. Based on PSNR test, proposed method obtained higher value for sample 3 and 5 as the more image noise appeared when more image details had been preserved. Without high amounts of tiny details, the proposed method can reduce more noises as compared to DCP.

Table 3. PSNR test

\begin{tabular}{l|rl}
\hline Sample & Proposed method & DCP \\
\hline 1 & 21.2968 & 20.4477 \\
2 & 21.4475 & 15.8471 \\
3 & 17.5691 & 54.9642 \\
4 & 18.0488 & 16.5846 \\
5 & 19.7013 & 20.2706 \\
\hline
\end{tabular}

\section{CONCLUSION}

The proposed method shows a good performance on underwater image enhancement as the image visibility range had been increased and preserve more image details. From the MSE test, the proposed method proved to recover the image contrast accurately. From the PSNR test, the proposed method had reduced more noise as compared with DCP if the image details are not too many. Overall, the proposed method had a good result on improving the image visibility range and preserving the image details. The future 
works of this paper will be colour correction on underwater image to avoid the bluish and greenish appearance.

\section{ACKNOWLEDGEMENT}

This study is supported by UMS Innovation Grant Scheme (SGI).

\section{REFERENCES}

Borkar, S. \& Bonde, S.V. 2016, Underwater image restoration using single color channel prior. 2016 International Conference on Signal and Information Processing (IConSIP), 1-4.

He, K., Sun, J. \& Tang, X. 2009, Single image haze removal using dark channel prior. IEEE Conference on Computer Vision and Pattern Recognition, 1956-1963.

Hitam, M.S., Yussof, W.N.J.H.W., Awalludin, E.A. \& Bachok, Z. 2013, Mixture contrast limited adaptive histogram equalization for underwater image enhancement. In proc. Of IEEE International Conference on Computer Applications Technology, 1-5.

Li, C., Guo, J., Pang, Y., Chen, S., \& Wang, J. 2016, Single underwater image restoration by blue-green channels dehazing and red channel correction. 2016 IEEE International Conference on Acoustics, Speech and Signal Processing (ICASSP), 1731-1735.

Lu, H., Li, Y. \& Serikawa, S. 2013, Underwater image enhancement using guided trigonometric bilateral filter and fast automatic color correction. 2013 IEEE International Conference on Image Processing, 3412-3416.

Sahtya, R., Bharathi, M. \& Dhivyasri, G. 2015, Underwater image enhancement by dark channel prior. IEEE Second International Conference on Electronics and Communication System (ICES 2015), 1119-1123.

Schechner, Y.Y. \& Karpel, N. 2005, Recovery of underwater visibility and structure by polarization analysis. IEEE Journal of Oceanic Engineering, 30, 3, 570-587.

Son, M., Lee, Y., Kang, H. \& Lee, S. 2014, Art-photographic detail enhancement. Computer Graphics Forum (Special Issue on Eurographics), 33, 2, 391-400.
Wen, H., Tian, Y., Huang, T. \& Gao, W. 2013, Single underwater image enhancement with a new optical model. 2013 IEEE International Symposium on Circuits and Systems (ISCAS), 753-756.

Wong, S.L., Yu, Y.P., Ho, A.J. \& Paramesran, R. 2014, Comparative analysis of underwater image enhancement methods in different color spaces. In Proc. of International Symposium on Intelligent Signal Processing and Communication Systems, 034-038.

Yang, H.Y., Chen, P.Y. \& Shiau, Y.H. 2011, Low complexity underwater image enhancement based on dark channel prior. 2011 Second Conference on Innovations in Bioinspired Computing and Applications (IBICA), 17-20.

Yao, B. \& Xiang, J. 2018, Underwater image dehazing using modified dark channel prior. 2-18 Chinese Control and Decision Conference (CCDC), 5792-5797.

Yao, D.N.Y., Bade, A., Suaib, N.M. \& Sulaiman, H, A. 2018, Digital image enhancement using enhanced detail and dehaze technique (DDE). Advanced Science Letters, 24, 3, 1559-1561.

Zheng, L., Shi, H. \& Sun, S. 2016, Underwater image enhancement algorithm based on CLAHE and USM. Proceedings of the IEEE International Conference on Information and Automation Ningbo, China, 585-590. 